\title{
Les récepteurs de l'angiotensine II dans le placenta humain sont de type $\mathrm{AT}_{1}$
}

\author{
MK Kalenga 1, *, M de Gasparo 2, R de Hertogh 1, \\ S Whitebread ${ }^{2}$, L Vankrieken ${ }^{1}, \mathrm{~K}$ Thomas 1 \\ 1 Université catholique de Louvain, département de gynécologie-obstétrique, \\ unité de recherche en physiologie de la reproduction humaine, \\ 5330, Avenue E Mounier, 1200 Bruxelles, Belgique; \\ 2 Laboratoire CIBA-GEIGY, Bâle, Suisse
}

(Reçu le 9 septembre 1990; accepté le 4 février 1991)

\begin{abstract}
Résumé - Les récepteurs membranaires d'angiotensine II ont été mesurés au niveau du placenta et des vaisseaux ombilicaux humains à l'aide de la ${ }^{125}$ [ $\mathrm{Sar}^{1}{ }^{\mathrm{He}}{ }^{8}$ ] All (antagoniste de l'angiotensine II) et ont été classés selon leur degré d'affinité vis-à-vis de 2 autres antagonistes de l'angiotensine II : DuP 753 et CGP 42112A. Le premier permet de déceler la présence du sous-type AT, et le second met en évidence le sous-type $A T_{2}$. La [Sar ${ }^{1} \mathrm{le}^{8}$ ] All a une affinité comparable $\left(\mathrm{K}_{\mathrm{d}} \approx 1 \mathrm{mmol} . \mathrm{I}^{-1}\right)$ dans les différentes structures placentaires examinées. Le $B_{\max }$ du tissu villositaire est semblable à celui de la plaque basale mais il est environ 9 fois plus élevé que celui de la plaque choriale. Dans la partie centrale du placenta, le nombre de sites récepteurs mesurés à une concentration de 0,15 nmol..$^{-1}$ de ${ }^{125}$ [ $\left[\mathrm{Sar}^{1} \mid \mathrm{ll} \mathrm{e}^{8}\right.$ ] All est en moyenne de $242 \pm 31 \mathrm{fmol} / \mathrm{mg}$ de protéines dans la plaque basale, $300 \pm 35$ dans les villosités choriales et $36 \pm 8$ dans la plaque choriale. La paroi de la veine et celle des artères du cordon ombilical contiennent respectivement $8,8 \pm 4,8$ et $4,0 \pm 1,7 \mathrm{fmol} / \mathrm{mg}$ de protéines. Dans les tissus placentaires, seul le sous-type AT, a été observé. Tenant compte des valeurs du $B_{\max }$ et du nombre de sites mesurés à une concentration unique de $0,15 \mathrm{nmol.} . \mathrm{I}^{-1}$ de ${ }^{125 \mid}$ $\left[\mathrm{Sar}^{1} \mathrm{Ile}^{8}\right]$ All, cette étude indique que les plus fortes concentrations des récepteurs de l'angiotensine II se situent dans les villosités choriales.
\end{abstract}

récepteur / angiotensine II / placenta humain / cordon ombilical

Summary - Human placenta contains only $\mathrm{AT}_{1}$ angiotensin II receptor subtype. Membrane angiotensin II receptors were measured in human placenta by means of ${ }^{125}$ / [Sar $1 / e^{8}$ ] All (angiotensin (I antagonist) and characterized by using 2 other antagonists of angiotensin II: Dup 753 and CGP 42112A. These are specific and selective ligands which enable identification of $A T_{1}$ and $A T_{2}$ receptor subtypes respectively. The [Sar $\left.{ }^{1} \| e^{8}\right]$ All affinity is similar $\left(K_{d}=1 \mathrm{nmol} . \mathrm{I}^{-1}\right)$ in the 3 different placental structures examined. However, the $\mathrm{B}_{\max }$ of villous tissues is $\approx 9$ times higher than that observed in chorionic plate but remains near that found in basal plate. In the central area of the placenta, mean values of ${ }^{125}$ [ $\left[\mathrm{Sar}^{1} \mathrm{Nl}^{8}\right.$ ] All binding observed at a single concentration of 0.15 $n$ mol..$^{-1}$ are $242 \pm 31 \mathrm{fmol} / \mathrm{mg}$ proteins in basal plate, $300 \pm 35$ in villous tissues and $36 \pm 8$ in chori-

\footnotetext{
* Correspondance et tirés à part
} 
onic plate. The umbilical vein and arteries respectively have $8.8 \pm 4.8$ and $4.0 \pm 1.7 \mathrm{fmol} / \mathrm{mg}$ protein. The subtype analysis shows that only $A T_{1}$ receptor is present in placental tissues. The $\mathrm{B}_{\text {max }}$ values as well as those obtained by the relative measurement performed at a fixed ${ }^{125}$ / [Sar $\|{ }^{1}{ }^{8}$ ] All concentration of 0.15 nmol..$^{-1}$ indicate that the highest concentrations of angiotensin II receptors are found in placental villous tissues.

receptor / antiotensin I/ / human placenta / umbilical cord

\section{INTRODUCTION}

Un certain nombre de travaux ont été consacrés à l'analyse du système rénineangiotensine au niveau des annexes fœtales. Le placenta, le cordon ombilical, le chorion et l'amnios contiennent, non seulement de la rénine, mais aussi de l'angiotensine I et de l'angiotensine II. Celle-ci est le composant le plus actif du système (Symonds et al, 1968; Poisner et al, 1981; Acker et al, 1982; Egan et al, 1988; Pinet et al, 1988; Lenz et al, 1989).

En effet, l'angiotensine II exerce plusieurs actions. Elle joue un rôle important dans la régulation de la pression artérielle grâce à son action vasopressive très puissante et grâce à sa participation au maintien de l'équilibre hydrosodé en stimulant la sécrétion de l'aldostérone et de la vasopressine (Albertini et al, 1980; Meyer, 1983; Alhenc-Gelas et al, 1985).

En outre, il a été démontré que l'angiotensine II stimule la sécrétion de l'hormone placentaire lactogène ( $\mathrm{hPL}$ ) par des cellules trophoblastiques (Petit et al, 1989), la sécrétion de la prolactine par des cellules adénohypophysaires (Platia et al, 1986; Myers et Steele, 1989) et la biosynthèse des hormones stéroïdes par les cellules de la granulosa (Pucell et al, 1987; Guichard et al, 1988; Palumbo et al, 1989). Dans cet esprit, l'on pense qu'en exerçant des actions diverses au niveau des annexes fœtales, l'angiotensine II pourrait contribuer efficacement au maintien de la grossesse et à la croissance fotale (Albertini et al, 1980; Alhenc-Gelas et al, 1985; Brar et al, 1986).

Les récepteurs d'angiotensine II ont été récemment observés dans le placenta (villosités choriales), le chorion et l'amnios (Cooke et al, 1981; Wilkes et al, 1985; Tencé et Petit, 1989; Kurauchi et al, 1990). Mais l'on n'a pas d'informations précises concernant leur classification et leur distribution dans ces tissus.

Dans ce travail, nous voulons mesurer les récepteurs membranaires de l'angiotensine II dans les différentes structures du placenta et dans les vaisseaux ombilicaux et ensuite tenter de définir leurs soustypes.

\section{MATÉRIEL ET MÉTHODES}

L'étude porte sur 8 placentas humains issus de grossesses suivies dans le service d'obstétrique des cliniques universitaires Saint-Luc (Bruxelles) et ayant évolué normalement jusqu'à terme. Aucune parturiente n'a présenté d'hypertension artérielle.

A l'accouchement, l'âge gestationnel moyen était de $38,50 \pm 1,73$ semaines avec des extrêmes allant de 37 à 41 semaines. Sept grossesses se sont terminées par un accouchement spontané et une grossesse par une césarienne pratiquée à cause d'une souffrance fœtale aigüe.

Immédiatement après son expulsion et son examen macroscopique en salle d'accouche- 
ment, le placenta est enveloppé dans un sachet en plastique et gardé momentanément au réfrigérateur à $4^{\circ} \mathrm{C}$ avant d'être transféré quelques min plus tard à l'unité de recherche en physiologie de la reproduction humaine. II est minutieusement lavé à l'aide de la solution physiologique ( $\mathrm{NaCl} 0,9 \%$ conservé à $4^{\circ} \mathrm{C}$ ) et ensuite déposé dans un bac à glace pour sa dissection.

Le cordon ombilical est disséqué aux ciseaux fins; la veine et les 2 artères ombilicales sont prélevées. Le placenta est libéré des membranes amniotique et chorionique. Quelques vaisseaux parcourant la face fœetale du placenta (ramifications de la veine et des artères ombilicales) sont également prélevés. Au centre et au bord de la masse placentaire, le prélèvement des tissus est fait sur la plaque choriale (face fœtale), sur la plaque basale (face maternelle) et sur les villosités choriales, masse se trouvant entre les 2 plaques.

Une fois prélevés, les échantillons des tissus sont rincés séparément avec la solution physiologique, placés chacun dans un tube en plastique et immédiatement congelés dans l'azote liquide. Ils sont aussiôt expédiés dans une boîte contenant de la neige carbonique, au laboratoire de CIBA-GEIGY (Bâle) pour la mesure des récepteurs membranaires d'angiotensine II. Celleci est réalisée selon une technique décrite en détail par Whitebread et al (1989).

Brièvement, le tissu est homogénéisé (Polytron, Kinematica, Lucerne, Suisse) dans 20 mmol. $\left.\right|^{-1} \mathrm{NaHCO}_{3}$ et centrifugé à $600 \mathrm{~g}$ et à $4^{\circ} \mathrm{C}$ pendant $20 \mathrm{~min}$. Le surnageant est à son tour centrifugé à $80000 \mathrm{~g}$ pendant $20 \mathrm{~min}$, le culot est suspendu dans $1 \mathrm{ml}$ de tampon Tris$\mathrm{HCl}\left(50 \mathrm{mmol} .1^{-1} \mathrm{pH} 7,4\right)$ contenant du $\mathrm{NaCl}$ (125 mmol. m $^{-1}, \mathrm{MgCl}_{2}\left(6,5 \mathrm{mmol}^{-1}\right)$, EDTA (1 mmol..$^{-1}$ ) et la concentration de protéines est mesurée selon la technique de Bradford (1976). La suspension de membranes est diluée à l'aide du même tampon pour avoir une concentration tissulaire en protéines comprise entre 62,5 et $250 \mu \mathrm{g} / \mathrm{ml}$. Ensuite, un cocktail d'inhibiteurs des peptidases à la concentration finale de $1 \mu \mathrm{g} / \mathrm{ml}$ et l'albumine de bœuf ( $2 \mathrm{mg} / \mathrm{ml}$ ) sont ajoutés à la suspension de membranes (Wilkes et al, 1985).

L'étude de la liaison de la [Sar $\left.{ }^{1} \mathrm{Ie}^{8}\right]$ All radio-iodée (Anawa, Wangen, Suisse) est réalisée dans un volume de $250 \mu$ l comprenant 200 $\mu l$ de membranes, $25 \mu l$ de traceur et $25 \mu l$ de l'agent compétiteur non marqué. Le mélange est incubé à $25^{\circ} \mathrm{C}$ pendant 60 min et la réaction est terminée par l'addition de tampon PBS glacé. La [Sar ${ }^{1} \mathrm{Ile}^{8}$ ] All radioactive liée aux membranes est séparée de la [Sar' ${ }^{1} \mathrm{Il}^{8}$ ] All libre sur un filtre Whatman GF/F (Filterprep 101, Ismatec, Zurich, Suisse). La radioactivité du filtre est comptée dans un spectromètre gamma (Pharmacia-LKB, Uppsala, Suède). La liaison maximale $\left(B_{\max }\right)$ et la constante de dissociation à l'équilibre $\left(K_{d}\right)$ sont étudiées dans quelques cas (courbes de compétition et graphiques selon Scatchard). Une mesure relative du nombre des sites récepteurs est effectuée dans tous les échantillons tissulaires à une concentration unique de 0,15 mmol..$^{-1}$ de ${ }^{125}$ [Sar ${ }^{1} \|^{8}$ ] All. La liaison non spécifique est déterminée en présence $d^{\prime} 1$ umol. $\left.\right|^{-1}$ d'angiotensine II non marquée et reste intérieure à $25 \%$. La [Sar ${ }^{1} \|^{8}$ ] All et l'angiotensine II froides proviennent de la firme Bachem (Bubendorf, Suisse).

Le CGP 42112A [nicotinic acid-Tyr-( $N^{\alpha}$ benzyloxycarbonyl-Arg)Lys-His-Pro-lle-OH] et le DuP 753 [2-butyl-4-chloro-1-[(2'-(1H-tétrazol-5yl)biphenyl-4-yl]méthyl]5-(hydroxyméthyl) imidazol] synthétisés par Ciba-Geigy (Bâle, Suisse) sont utilisés pour différencier les sous-types du récepteur de l'angiotensine II. Selon le travail de Whitebread et al (1989) et celui de Rogg et al (1990), le CGP 42112A a une plus grande affinité pour le sous-type $A T_{2}\left(K_{d}=0,5 \mathrm{nmol} . \mathrm{l}^{-1}\right)$ que pour le sous-type $A T_{1}\left(K_{d}=3 \mu \mathrm{mol}^{-1} \mathrm{I}^{-1}\right)$. L'affinité du Dup 753 est plus de 1000 fois plus élevée pour le sous-type $A T_{1}\left(K_{d}=31 \mathrm{nmol}^{-1}\right)$ que pour le sous-type $\left.A T_{2}\left(K_{d}=33 \mu \mathrm{mol}^{-1}\right)^{-1}\right)$.

Le programme LIGAND est employé pour l'analyse des courbes de compétition (Munson et Rodbard, 1980; Whitebread et al, 1989) et le test de Student pour l'étude comparative du nombre des sites.

L'examen histologique de diverses structures placentaires obtenues après dissection ( $n=5$ pour chacune d'elles) est pratiqué dans le but de vérifier l'homogénéité de celles-ci. II résulte de cet examen que la contamination des échantillons de la plaque choriale par le tissu villositaire et vice-versa est de moins de 5\% tandis que dans les échantillons de la plaque basale, l'on retrouve $50 \%$ du tissu villositaire. Celui-ci est contaminé par la plaque basale dans une proportion de moins de $5 \%$. 


\section{RÉSULTATS}

Le nombre de sites récepteurs est exprimé en $\mathrm{fmol} / \mathrm{mg}$ protéines. Notons qu'il existe une bonne corrélation entre la liaison spécifique de la ${ }^{125}$ [Sar ${ }^{-1} \| e^{8}$ ] All et la concentration tissulaire des protéines. Audelà de $250 \mu \mathrm{g}$ de protéines $/ \mathrm{ml}$ la relation linéaire tend à disparaître (fig 1).

Les courbes de compétition réalisées sur lo tissu placentaire (fig 2) nous montrent le degré d'inhibition des récepteurs d'angiotensine II par les antagonistes de l'angiotensine II. La [Sar ${ }^{1} \mathrm{He}^{8}$ ] All dont $I^{\prime} I C_{50}$ varie entre 1,25 et $1,61 \mathrm{nmol} . I^{-1}$ (tableau I) inhibe la totalité des sites. Le Dup 753 qui exerce un effet inhibiteur sélectif sur le sous-type $A T_{1}$, inhibe également la totalité des sites $\left(\mathrm{IC}_{50}\right.$ entre 33,0 et 38,1 nmol.1-1). Aux faibles concentrations (nmol. (1) $^{-1}$ le CGP 42112A n'a aucun effet. À des concentrations micromolaires, il inhibe tous les sites $\left(\mathrm{IC}_{50}\right.$ entre 1,31 et 3,78 $\mu \mathrm{mol} . \mathrm{I}^{-1}$ ) comme démontré précédemment sur les cellules musculaires lisses de l'aorte du rat mises en culture (Whitebread et al, 1989). Ces résultats suggèrent que les tissus placentaires examinés ne contiennent que le sous-type $\mathrm{AT}_{1}$.

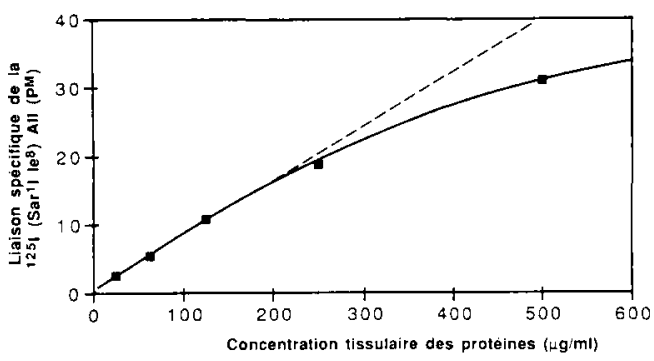

Fig 1. Corrélation entre la liaison spécifique de la ${ }^{125}$ [ [Sar $\left.{ }^{1} \| e^{8}\right] A l l$ et la concentration des protéines dans le tissu villositaire d'un placenta humain à terme.

Sur la représentation graphique selon Scatchard (fig 3) et le tableau II, le $B_{\max }$ de la plaque choriale est de $215 \mathrm{fmol} / \mathrm{mg}$ protéines mais celui des villostés choriales est environ 8-12 fois plus élevé, variant entre 1641 et $2582 \mathrm{fmol} / \mathrm{mg}$ protéines. La plaque basale a un $B_{\max }$ de $1680 \mathrm{fmol} / \mathrm{mg}$ protéines au centre et 2196 au bord du placenta. Dans les 3 structures du placenta, les valeurs du $K_{d}$ sont de l'ordre de 1 nmol. I $^{-1}$. Sur les vaisseaux du cordon ombilical et ceux de la face fœtale du placenta, le $B_{\max }$ et le $K_{d}$ n'ont pas été déterminés car la liaison spécifique y est extrêmement faible.

Tableau I. L'IC $_{50}$ (en nmol. I-1) pour la [Sar $\left.{ }^{1} \| e^{8}\right] A l l$, le CGP $42112 A$ et le Dup 753 dans les différentes structures d'un placenta humain à terme.

\begin{tabular}{llll}
\hline Localisation & [Sar ${ }^{\left.1 / l e^{8}\right] A \| l}$ & Dup 753 & CGP 42112A \\
\hline Plaque choriale & 1,61 & 33,0 & 1310 \\
Plaque basale & 1,25 & 38,1 & 3780 \\
Villosités choriales & 1,3 & 35,2 & 1920 \\
\hline
\end{tabular}

Fig 2. Inhibition de la liaison de la ${ }^{125}$ [Sar $\left.{ }^{1} \| e^{8}\right] A l l$ aux récepteurs du placenta humain par la [Sar'1le]]All froide, le Dup 753 (inhibiteur du sous-type AT ${ }_{1}$ ) et le CGP 42112A. Celui-ci est un inhibiteur sélectif du sous-type AT 2 mais aux plus fortes concentrations, il inhibe les 2 sous-types. $d=$ plaque choriale $(250 \mu \mathrm{g}$ de protéines $/ \mathrm{ml})$, e $=$ plaque basale $(62,5 \mu \mathrm{g}$ de protéines $/ \mathrm{ml}), \mathrm{f}=$ villosités choriales $(62,5 \mu \mathrm{g}$ de protéines $/ \mathrm{ml})$. Les tissus placentaires ont été prélevés à l'issue d'une grossesse à terme. 
Récepteurs d'angiotensine II dans le placenta humain
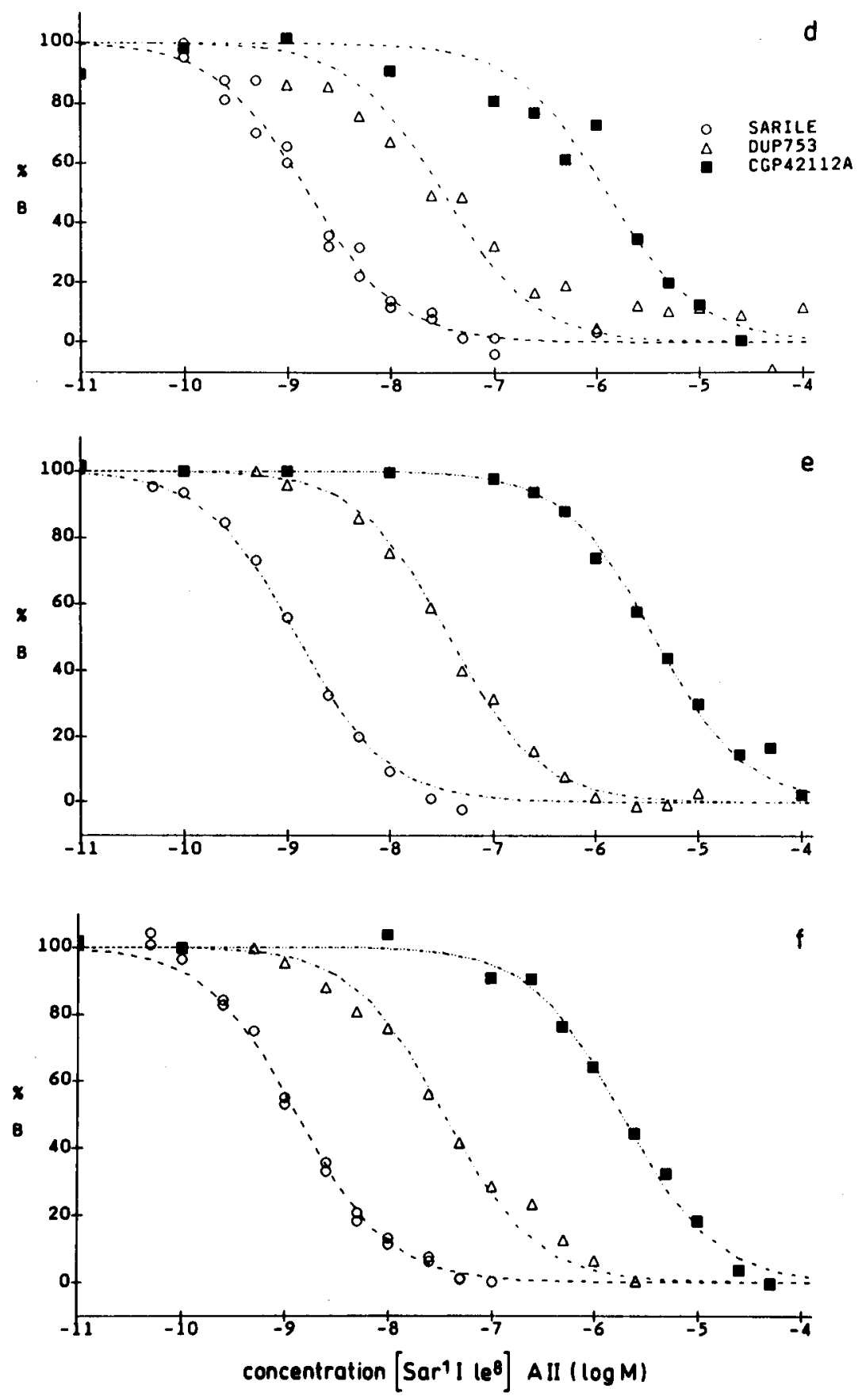

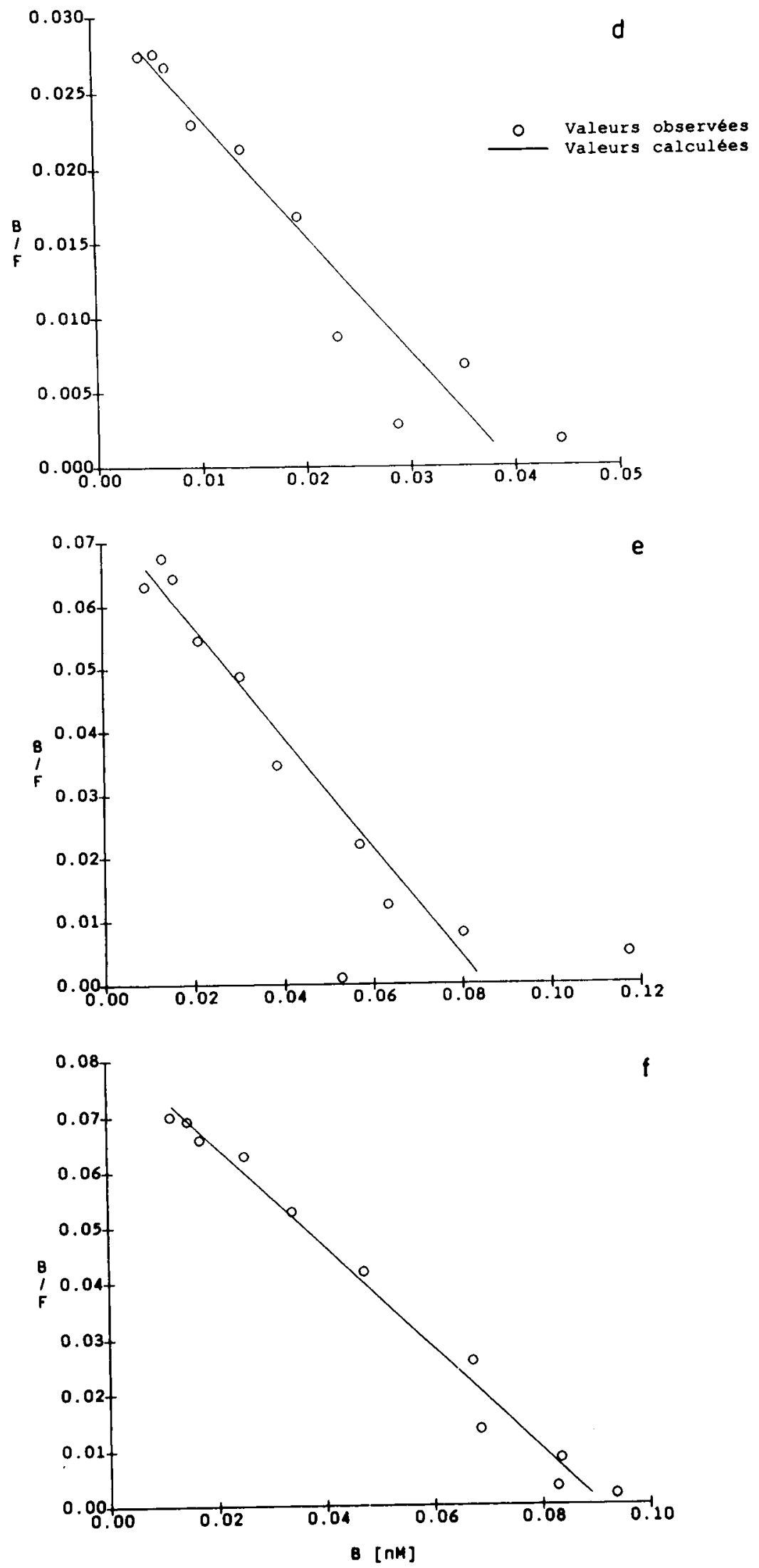
Considérant que l'affinité de la [Sar' $\left.\mathrm{Ile}^{8}\right]$ All est similaire dans la plaque choriale, la plaque basale et les villosités choriales, nous avons mesuré la proportion relative des récepteurs en n'utilisant qu'une seule concentration de traceur radioactif. À une concentration de $0,15 \mathrm{nmol}^{-1} \mathrm{l}^{-1}$ de 125 | [Sar $\left.{ }^{1} \mathrm{Ile}^{8}\right]$ All, le nombre des sites occupés est en moyenne de $36 \pm 8 \mathrm{fmol} / \mathrm{mg}$ de protéines dans la plaque choriale (fig 4 ) : il est 5-6 fois plus grand que celui qui est observé dans les vaisseaux du cordon ombilical et des vaisseaux de la face foetale du placenta. La plaque basale et les villosités choriales ont des concentrations relativement plus élevées atteignant respectivement en moyenne $242 \pm 31$ et $300 \pm 35$ $\mathrm{fmol} / \mathrm{mg}$ de protéines dans la partie centrale, $250 \pm 32$ et $343 \pm 32$ au bord de la masse placentaire.

La comparaison des valeurs moyennes ne montre pas de différence significative entre la plaque basale et les villosités choriales mais dans chacune de ces 2 structures, le nombre des sites occupés est significativement $(P<0,001)$ plus élevé que celui observé dans la plaque choriale. Dans une même structure, la proportion relative des sites récepteurs est comparable au centre et au bord de la masse placentaire (fig 4).

\section{DISCUSSION}

La mesure de récepteurs membranaires d'angiotensine II au niveau du placenta et des vaisseaux ombilicaux humains a été réalisée à l'aide d'une technique antérieurement décrite par Whitebread et al (1989). Cette méthode a été testée sur plusieurs tissus de diverses espèces ani-

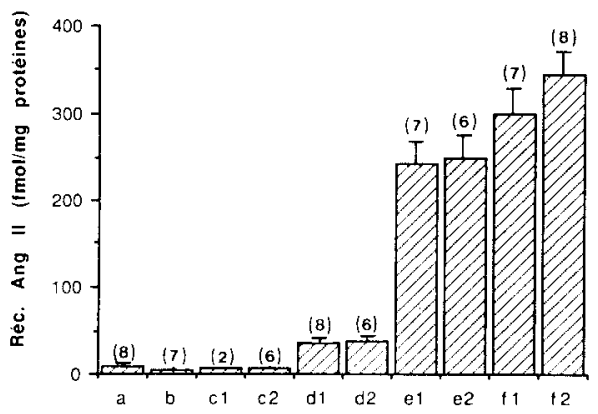

Fig 4. Mesure relative des récepteurs de l'angiotensine II (valeurs moyennes + erreur standard) au niveau du placenta et des vaisseaux ombilicaux humains, à une concentration fixe de 0,15 nmol..$^{-1}$ de ${ }^{125}\left[{ }^{2} \operatorname{Sar}^{1} \| e^{8}\right]$ All. Les tissus proviennent des grossesses à terme. $a=$ veine ombilicale, $b=$ artères ombilicales, $c_{1}=$ veines/face fœtale du placenta, $c_{2}=$ artères/face fœtale du placenta, $d=$ plaque choriale prélevée au centre $\left(d_{1}\right)$ et au bord $\left(d_{2}\right)$ du disque placentaire, e = plaque basale prélevée au centre $\left(e_{1}\right)$ et au bord $\left(e_{2}\right)$ du disque placentaire, $f=$ villosités choriales prélevées au centre $\left(f_{1}\right)$ et au bord $\left(f_{2}\right)$ du disque placentaire. Les chiffres entre parenthèses représentent le nombre de cas.

males et a permis de découvrir et de caractériser 2 classes de récepteurs de l'angiotensine II provisoirement appelés $A$ et B. Il a été démontré notamment chez la ratte que les 2 sous-types existent au niveau de la zone glomérulaire surrénalienne et au niveau du muscle utérin. Dans l'espèce humaine, la zone glomérulaire de la glande surrénale possède également les 2 sous-types, mais le myomètre ne contient que le récepteur $\mathrm{A}$ (Whitebread et al, 1989).

Une certaine confusion existe dans la littérature (Chiu et al, 1989; Chang et Lotti,

Fig 3. Représentation graphique selon Scatchard du rapport entre les concentrations de [Sar $\left.{ }^{1} \mid l e^{8}\right] A l l$ liée $(B)$ et libre $(F)$ exprimé en fonction des concentrations de [Sar $\left.{ }^{1} l l e^{8}\right]$ All liée aux récepteurs du placenta humain à terme. $\mathrm{d}=$ plaque choriale $(250 \mu \mathrm{g}$ de protéines $/ \mathrm{ml}), \mathrm{e}=$ plaque basale $(62,5 \mu \mathrm{g}$ de protéines $/ \mathrm{ml}), \mathrm{f}=$ villosités choriales $(62,5 \mu \mathrm{g}$ de protéines $/ \mathrm{ml})$. 
Tableau II. $B_{\max }$ et $K_{d}$ observés dans la plaque choriale, la plaque basale et les villosités choriales du placenta humain à terme. La [Sar $\left.{ }^{1} l e^{8}\right] A$ All est utilisée comme radioligand.

\begin{tabular}{lll}
\hline Localisation & $B_{\max }$ (fmol/mg protéines) & $K_{d}\left(n\right.$ mol. $\left.\mathrm{H}^{-1}\right)$ \\
\hline Plaque choriale $\left(d_{1}\right)$ & 215 & 1,3 \\
Plaque basale & & \\
$\mathrm{e}_{1}$ & 1680 & 1,1 \\
$\mathrm{e}_{2}$ & 2196 & 1,4 \\
Villosités choriales $_{01 f_{1}}$ & & \\
$02 \mathrm{f}_{1}$ & 2104 & 1,3 \\
$06 \mathrm{f}_{1}$ & 1741 & 1,2 \\
$01 \mathrm{f}_{2}$ & 1641 & 1,7 \\
$02 \mathrm{f}_{2}$ & 1820 & 1,1 \\
$06 \mathrm{f}_{2}$ & 2151 & 0,9 \\
& 2582 & 1,0 \\
\hline
\end{tabular}

$d_{1}=$ Plaque choriale prélevée au centre du disque placentaire; $e=$ plaque basale au centre $\left(\theta_{1}\right)$ et au bord $\left(e_{2}\right)$ du disque placentaire; $f=$ villosités choriales au centre $\left(f_{1}\right)$ et au bord $\left(f_{2}\right)$ du disque placentaire.

1990; Speth et Kim, 1990) à cause d'une nomenclature différente des mêmes soustypes. Ainsi, Chiu et al (1989) ont confirmé l'existence de 2 sous-types de récepteurs en utilisant 2 antagonistes non peptidiques de l'angiotensine II dans une expérience menée sur des rats. Le sous-type 1 (correspondant au sous-type $B$ ) est révélé par le "DuP 753 " et représente $80 \%$ des sites du cortex surrénalien. Le sous-type 2 (identique au sous-type A) est mis en évidence par le “EXP 655», un antagoniste non peptidique différent du CGP 42112A et se trouve en grandes concentrations dans la zone médullaire de la surrénale où il atteint $90 \%$ du total des sites.

Une commission internationale a proposé d'appeler le sous-type sensible au DUP 753 et celui sensible au CGP 42112A, respectivement $A T_{1}$ et $A T_{2}$ (Bumpus et al,
1991). Depuis lors, cette nomemclature est utilisée.

Sur le plan fonctionnel, Bottari et al (1990) ont constaté que les 2 sous-types se comportent différemment en présence de l'angiotensine II : le récepteur $A T_{1}$ se lie à une G-protéine (guanine nucleotidebinding-protein) et forme un complexe avec celle-ci alors que le récepteur $\mathrm{AT}_{2}$ reste non couplé. D'autres études indiquent que le récepteur $A T_{1}$ est responsable de la contraction vasculaire produite par l'angiotensine II (Criscione et al, 1990; de Gasparo et al, 1990).

Dans le présent travail, nous notons qu'il existe, au niveau du placenta humain, un nombre important de récepteurs de l'angiotensine II. L'affinité de la [Sar' $\left.\|^{1} e^{8}\right]$ All pour ces récepteurs est semblable dans les 3 structures placentaires qui ont été 
examinées. L'analyse du $B_{\max }$ et du nombre de sites mesurés à une concentration de $0,15 \mathrm{nmol}^{-I^{-1}}$ de ${ }^{125}$ [Sar ${ }^{1} \mathrm{Ie}^{8}$ ] All montre que les villosités choriales contiennent des concentrations de récepteurs les plus élevées. Les vaisseaux du cordon ombilical et ceux de la face fotale du placenta lient très faiblement la [Sar $\left.{ }^{1} \mathrm{He}^{8}\right]$ All.

Le Scatchard réalisé sur la fixation de la [Sar $\left.{ }^{1} \|^{8}\right]$ All découvre une seule classe de sites spécifiques dans la plaque choriale, la plaque basale et dans les villosités choriales (fig 2). Le Dup 753 nous a permis, grâce à son affinité sélective, de préciser que cette classe de récepteurs présents dans le tissu placentaire appartient au sous-type $A T_{1}$. Cette observation corrobore les résultats de Whitebread et al (1989), Chiu et al (1989); Chang et Lotti (1990) et ceux de Speth et Kim (1990), montrant bien que pour la classification des récepteurs, l'utilisation des antagonistes de l'angiotensine II est mieux indiquée que celle de l'angiotensine II ellemême.

Quelques études antérieures concernant les récepteurs d'angiotensine II dans les villosités choriales (Cooke et al, 1981; Wilkes et al, 1985; Tencé et Petit, 1989; Kurauchi et al, 1990) ont révélé, sur base de l'analyse du Scatchard, une seule classe de récepteurs mais le typage de ces derniers n'a pas été fait. Le $B_{\max }$ et le $\mathrm{K}_{d}$ observés sur la liaison de l'angiotensine Il varient d'une étude à l'autre et cela, probablement en fonction de la méthode appliquée : des valeurs extrêmes vont pour le $B_{\max }$ de 38,40 à $1500 \mathrm{fmol} / \mathrm{mg}$ protéines et pour le $K_{d}$ de 0,27 à $8,97 \mathrm{nmol}^{-1}{ }^{-1}$. Les valeurs du $B_{\max }$ que nous avons obtenues à ce sujet sont à la limite supérieure des chiffres qui ont été publiés dans la littérature. Ceci pourrait être lié au fait que nous avons utilisé comme traceur radioactif, la [Sar ${ }^{1} \| e^{8}$ ] All qui est un antagoniste de l'angiotensine II, plus stable que celle-ci.
En conclusion, les résultats de cette étude montrent de manière originale que les récepteurs de l'angiotensine II observés au niveau du placenta humain appartiennent au sous-type $A_{1}$ et que le plus grand nombre de ces récepteurs ( $B_{\max }$ et sites de liaison mesurés à une concentration de $0,15 \mathrm{nmol}^{-1} \mathrm{I}^{-1}$ de ${ }^{125}\left[\mathrm{Sar}^{1} \mathrm{Ile}^{8}\right.$ ] All) se trouvent dans les villosités choriales. L'angiotensine II pourrait dès lors jouer un rôle important dans la régulation de la fonction placentaire.

\section{REMERCIEMENTS}

Nous remercions sincèrement les infirmières de la salle d'accouchement des cliniques universitaires Saint-Luc (Bruxelles) et Y Bogdale (CIBAGEIGY, Bâle) pour leur contribution à la réalisation de ce travail.

Nos remerciements s'adressent également à $J$ Rahier et $S$ Gosseye pour l'analyse des coupes histologiques et à $A$ Kalenga pour sa participation à la préparation du manuscrit.

\section{RÉFÉRENCES}

Acker GM, Galen FX, Devaux C, Foote S, Papernik E, Pesty A, Menard J, Corvol P (1982) Human chorionic cells in primary culture: a model for renin biosynthesis. J Clin Endocrinol \& Metab 55, 902-909

Albertini R, Seiro M, Scicli AG, Carretero OA (1980) Uteroplacental renin in regulation of blood pressure in the pregnant rabbit. $A m \mathrm{~J}$ Physiol 239, 266-271

Alhenc-Gelas $F$, Tache A, Saint-André JP, Milliez J, Sureau $C$, Corvol $P$, Ménard $J$ (1985) Le système rénine-angiotensine au cours de la grossesse et de l'accouchement. In : Actualités néphrologiques de l'Hôpital Necker. Flammarion Médecine Science, Paris, 27-34

Bottari SP, Mele M, Taylor V, Whitebread S, de Gasparo M (1990) A subtype of angiotensin II receptors does not interact with guanine 
nucleotide regulatory proteins. 77 th Annu Meet Endocr Soc, Atlanta, June 20-23rd

Bradford MM (1976) A rapid and sensitive method for the quantitation of microgram quantities of protein utilizing the principle of protein-dye binding. Anal Biochem 72, 248-254

Brar HS, Do YS, Tam HB, Valenzuela GJ, Murray $R D$, Longo $L D$, Yonekura $M L$, Hsueh WA (1986) Uteroplacental unit as a source of elevated circulating prorenin levels in normal pregnancy. Am J Obstet Gynecol 155, 12231226

Bumpus FM, Catt KJ, Chiu AT, de Gasparo M, Goodfriend A, Husain A, Peach MJ, Taylor $V$, Timmermans PBMWM (1991) Nomenclature for angiotensin receptors. Hypertension (in press)

Chang RSL, Lotti VJ (1990) Two distinct angiotensin $I I$ receptor binding sites in rat adrenal revealed by new selective nonpeptide ligands. J Molecular Pharmacol 37, 347-351

Chiu AT, Herbin WF, MCCall DE, Ardecky RJ, Carini DJ, Duncia JV, Pease LJ, Wong PC, Wexler RR, Johnson AL, Timmermans PBMWM (1989) Identification of angiotensin II receptor subtypes. Biochem Biophys Res Commun 165, 196-203

Cooke SF, Graven DJ, Symonds EM (1981) A study of angiotensin II binding sites in human placenta, chorion and amnion. Am J Obstet Gynecol 140, 689-692

Criscione L, Thomann $H$, Whitebread $S$, de Gasparo M, Buehimayer P, Herold P, Ostermayer F, Kamber B (1990) Binding characteristics and vascular effets of various angiotensin 11 antagonists. J Cardiovasc Pharmacol $16,56-59$

de Gasparo M, Whitebread S, Mele M, Motani AS, Whitcombe PJ, Ramjoué HP, Kamber B (1990) Biochemical characterization of two angiotensin II receptor subtypes in the rat. $J$ Cardiovasc Pharmacol 16, 31-35

Egan DA, Grzegorczyk V, Tricarico KA, Rueter A, Holleman WH, Marcotte PA (1988) Human placental chorionic renin: production, purification and characterization. Biochim Biophys Acta 965, 68-75

Guichard A, Mignot TM, Boyer P, Zorn JR, Cédard $L$ (1988) Dosage immunoradiométrique de la rénine active et de la prorénine plasma- tiques au cours des cycles stimulés pour Fivete ou Gift. Reprod Nutr Dév 28, 1731-1740

Kurauchi $O$, Mizutani S, Nomura $S$, Kasugai M, Narita O, Tomoda $Y$ (1990) A comparison of the binding of angiotensin II to human placenta between normal and severe preeclamptic pregnancy. J Med 21, 209-216

Lenz T, Sealey JE, August $P$, James GD, Laragh $\mathrm{JH}$ (1989) Tissue levels of active and total renin, angiotensinogen, human chorionic gonadotropin, estradiol and progesterone in human placentas from different methods of delivery. J Clin Endocrinol Metab 69, 31-37

Meyer P (1983) Physiologie humaine. Flammarion Médecine-Sciences, Paris, 794-801

Munson PJ, Rodbard D (1980) Ligand: a versatile computerized approach for characterization of ligand-binding systems. Anal Biochem 107, 220-239

Myers LS, Steele MK (1989) The brain reninangiotensin system and the regulation of prolactin secretion in female rats: influence of ovarian hormones. $J$ Neuroendocrinology 1 , 299-303

Palumbo A, Jones $\mathrm{C}$, Lightman $\mathrm{A}$, Carcangiu ML, Decherney AH, Naftolin F (1989) Immunohistochemical localization of renin and angiotensin II in human ovaries. Am J Obstet Gynecol 160, 8-14

Petit A, Guillon G, Tencé M, Jard S, Gallo-Payet N, Bellabarba D, Lehoux JG, Belisle S (1989) Angiotensin II stimulates both inositol phosphate production and human placental lactogen release from human trophoblastic cells. $J$ Clin Endocrinol Metab 69, 280-286

Pinet F, Corvol MT, Bourguignon J, Corvol $P$ (1988) Isolation and characterization of reninproducing human chorionic cells in culture. $J$ Clin Endocrinol Metab 67, 1211-1220

Platia MP, Catt KJ, Aguilera G (1986) Effects of 17 beta-estradiol on angiotensin II receptors and prolactin release in cultured pituitary cells. Endocrinology 119, 2768-2772

Poisner AM, Wood GW, Poisner R, Inagami T (1981) Localization of renin in trophoblasts in human chorion laeve at term pregnancy. Endocrinology 109, 1150-1155

Pucell AG, Bumpus FM, Husain A (1987) Rat ovarian angiotensin II receptors characterization and coupling to estrogen secretion. $J$ Biol Chem 262, 7076-7080 
Rogg H, Schmid A, de Gasparo M (1990) Identjfication and characterization of angiotensin II receptor subtypes in rabbit ventricular myocardium. Biochem Biophys Res Commun (in press)

Speth RC, Kim KH (1990) Discrimination of 2 angiotensin II receptor subtypes with a selective agonist analogue of angiotensin II paraaminophenylalanine 6 angiotensin 11. Biochem Biophys Res Commun 169, 997-1006

Symonds EM, Stanley MA, Skinner SZ (1968) Production of renin by in vitro cultures of human chorion and uterine muscle. Nature (Lond) 217, 1152-1153
Symonds EM (1988) Renin and reproduction. Am J Obstet Gynecol 158, 754-761

Tencé M, Petit A (1989) Characterization of angiotensin II binding sites in the human term placenta. Mol Cell Endocrinol 63, 111-119

Wilkes BM, Krim E, Mento PF (1985) Evidence for a functional renin-angiotensin system in full-term fetoplacental unit. Am J Physiol 249, 366-373

Whitebread S, Mele M, Kamber B, De Gasparo M (1989) Preliminary biochemical characterization of two angiotensin II receptor subtypes. Biochem Biophys Res Commun 163, 284-291 